\title{
Prospective cardiovascular magnetic resonance imaging in adults with Alström syndrome: silent progression of diffuse interstitial fibrosis
}

Shanat Baig ${ }^{1,2}$, Rory Dowd ${ }^{3}$, Nicola C. Edwards ${ }^{2,3}$, James Hodson 4 , Larissa Fabritz ${ }^{2,3}$, Ravi Vijapurapu ${ }^{1,2}$, Boyang Liu ${ }^{1,2}$, Tarekegn Geberhiwot ${ }^{1,5}$ and Richard P. Steeds ${ }^{2,3,6^{*}}$ (i)

\begin{abstract}
Background: Alström syndrome (ALMS) is a rare ciliopathy characterised by early onset insulin resistance, obesity, and dyslipidaemia and is a model for diseases that have huge social, health and economic impact. Cardiomyopathy develops in the majority, with high rates of morbidity and mortality, the definitive features of which are coarse replacement fibrosis and diffuse myocardial fibrosis (DIF). The pathogenesis of heart failure is thought to involve fibroblast accumulation and expansion of the extracellular matrix with excess protein deposition, leading to distorted organ architecture and impaired contractile function. Consecutive adults with genetically proven ALMS attending the National Centre for Rare Disease in Birmingham, England were studied. All patients underwent serial CMR, echocardiography and venous blood sampling, with computed tomography coronary angiography (CTCA) performed to assess severity of CAD. The aims of this study were: 1) to evaluate changes over time in DIF by cardiovascular magnetic resonance tissue characterization in ALMS; 2) to examine whether changes in DIF are associated with alteration in systolic or diastolic function; and 3) to evaluate the frequency and severity of coronary artery disease as a confounder for progression of ischaemic versus non-ischaemic fibrosis.
\end{abstract}

Results: In total, 30/32 adults (63\% male; 67\% White British) participated. The median age at first scan was 21.3 years (interquartile range: 19.0-32.6) and participants were followed for a maximum of 67 months. Only 4 patients had significant coronary artery stenosis on post-mortem, invasive coronary angiography or CTCA. Mid short axis myocardial T1 times, myocardial extracellular volume, and left ventricular mass increased significantly over time, by an average of $21.8 \mathrm{~ms}(95 \% \mathrm{Cl} 17.4-26.1 ; p<0.001)$, 1.1 percentage points $(0.6-1.6, p<0.001)$, and $2.8 \mathrm{~g} / \mathrm{m}^{2}(1.9-3.7$; $p<0.001)$ per year, respectively. These changes were not associated with significant deterioration in myocardial structure or function.

\footnotetext{
*Correspondence: Rick.Steeds@uhb.nhs.uk

${ }^{2}$ Institute of Cardiovascular Science, University of Birmingham, Birmingham, UK

${ }^{3}$ Department of Cardiology, Queen Elizabeth Hospital, Birmingham, UK

Full list of author information is available at the end of the article
}

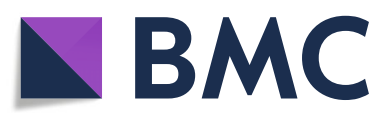

( The Author(s). 2020 Open Access This article is licensed under a Creative Commons Attribution 4.0 International License, which permits use, sharing, adaptation, distribution and reproduction in any medium or format, as long as you give appropriate credit to the original author(s) and the source, provide a link to the Creative Commons licence, and indicate if changes were made. The images or other third party material in this article are included in the article's Creative Commons licence, unless indicated otherwise in a credit line to the material. If material is not included in the article's Creative Commons licence and your intended use is not permitted by statutory regulation or exceeds the permitted use, you will need to obtain permission directly from the copyright holder. To view a copy of this licence, visit http://creativecommons.org/licenses/by/4.0/. The Creative Commons Public Domain Dedication waiver (http://creativecommons.org/publicdomain/zero/1.0/) applies to the data made available in this article, unless otherwise stated in a credit line to the data. 
(Continued from previous page)

Conclusions: This is the first comprehensive prospective study demonstrating progression of DIF in ALMS over time, although no structural or functional consequences were noted within a median three and a half years' follow up. Further study is warranted to define whether DIF is a by-stander or the driver to impaired contractile function, heart failure and death.

Keywords: Alström syndrome, Cardiovascular magnetic resonance, Diffuse interstitial fibrosis, Progression, Extracellular volume, Ischaemic heart disease

\section{Introduction}

Alström syndrome (ALMS) is a rare autosomal recessive cardiomyopathy (OMIM 203800) characterised by multiorgan disease. Infantile cardiomyopathy and retinal cone rod dystrophy are the earliest and most frequent manifestations of the syndrome. The most severe and often lethal complication of ALMS is heart failure due to cardiomyopathy [1]. Cardiomyopathy affects up to $65 \%$ of adolescents and adults, with high rates of morbidity and mortality [2]. Both the quality and length of life are reduced in adults with ALMS, and few survive beyond the age of 50 [2]. Autopsy data have demonstrated replacement myocardial fibrosis in non-coronary artery patterns, and diffuse interstitial fibrosis (DIF) has been detected on cardiovascular magnetic resonance (CMR) by elevation in T1 relaxation [3] and increased extracellular volume (ECV) in cross-sectional studies of ALMS [4]. These changes were associated with sub-clinical impairment of left ventricular (LV) function, assessed through change in myocardial strain. In many cardiovascular diseases, it has been postulated that the development of DIF over time may be a driver of abnormal myocardial deformation and diastolic dysfunction, leading ultimately to systolic heart failure [5]. While ALMS is rare, it offers a model of myocardial disease in which disease progression may be rapid, although serial assessment has not previously been performed.

Given the frequency of obesity, insulin resistance and metabolic syndrome in ALMS, ischaemic heart disease may well represent a potential confounder to increased T1 relaxation times and ECV [6]. Despite the high burden of $\mathrm{CV}$ risk factors, reported cases of coronary artery disease $(C A D)$ are rare and data have been limited [7]. Moreover, defining serial changes in T1 and ECV relative to the frequency of coronary disease is important on a clinical basis, since primary and secondary treatment strategies differ. Therefore, the aims of this study were: 1) to evaluate changes in DIF over time by CMR tissue characterization in adults with ALMS; 2) to examine whether changes in DIF could be associated with alteration in systolic or diastolic function; and 3) to evaluate the frequency and severity of coronary artery disease as a confounder for progression of ischaemic versus nonischaemic fibrosis.

\section{Methods}

\section{Study design}

Between March 2012 and January 2018, 32 consecutive adults with genetically proven ALMS attending the $\mathrm{Na}$ tional Centre for Alstrom syndrome at the Queen Elizabeth Hospital Birmingham, United Kingdom (UK) were evaluated as part of standard clinical care. The clinic is unusual in that it is patient-centred, run in association with the charity Alström Syndrome UK and involves physicians from different specialties, nurses and relevant professions allied to medicine, forming a truly multidisciplinary team (MDT). Patients attend for 2 days and in this time, undergo all their relevant investigations, and are seen by all members of the MDT. At the first and subsequent visit, patients underwent annual disease monitoring following a standardized clinical protocol, including: biochemistry, echocardiography, and CMR. This was an observational, retrospective audit, utilizing existing data collected as part of clinical service. It was approved by local clinical governance committees (CARMS-15674) and conformed to the principles of Good Clinical Practice guidelines. Patients were eligible for inclusion in this audit if they were $\geq 16$ years of age; had a genetically confirmed diagnosis of ALMS; and had been followed up in the service for a minimum of 1 year. Patients were excluded if they had an absolute contraindication to CMR (1 patient with implantable cardio defibrillator) or a relative contra-indication for the study (1 patient was on a research drug trial). Otherwise, all adult patients in the service were included. Serial CMR and single computed tomography coronary angiography (CTCA) were included as standard for ALMS patients. Annual CMR was instituted from the start of the clinical service, while CTCA was introduced soon after the incidental finding of an occluded right coronary artery in an asymptomatic 38 year old male.

\section{Investigations \\ Blood markers}

Venous blood samples were collected for haematology, biochemistry, and lipid profiles. Serum N-terminal pro B natriuretic peptide (NT-proBNP, ng/L) was measured by sandwich immunoassay with magnetic particle separation and chemiluminescent detection on an E170 
analyser (Roche Diagnostics, Burgess Hill, United Kingdom) with a lower limit of detection of $0.6 \mathrm{pmol} / \mathrm{L}$. Dyslipidaemia was defined as fasting cholesterol $>5 \mathrm{mmol} / \mathrm{L}$ and/or triglycerides $>1.7 \mathrm{mmol} / \mathrm{L}$.

\section{Echocardiography}

Resting transthoracic echocardiography (TTE; ie33, Phillips) was performed by an accredited sonographer according to the British Society of Echocardiography minimum dataset [8]. Diastolic function was graded by an experienced cardiologist specialising in echocardiography (RPS) according to current guidelines [9].

\section{Cardiac magnetic resonance imaging}

Contrast enhanced CMR (1.5 T Avanto, Siemens Healthcare, Erlangen, Germany) was performed in-line with standard protocols to obtain LV and right ventricular (RV) dimensions, volumes, and mass on a bi-annual basis [10]. A steady-state free precession, single breath hold modified Look-Locker inversion recovery (MOLLI) sequence was used for T1 mapping in the basal and mid left ventricular short axis levels and the horizontal longaxis, before and 15-20 min after administration of gadolinium-based contrast agent (GBCA) [10]. Average scan parameters: $8 \mathrm{~mm}$ slice with a 192 read-out matrix, $6 / 8$ phase partial Fourier with $81 \%$ phase resolution, field-of-view $320 \times 320 \mathrm{~mm}^{2}$, repetition time $2.4 \mathrm{~ms}$, echo time $1.01 \mathrm{~ms}, 11$ phases [3, 3, 5 scheme], total breath hold 17 R-R intervals [11]. LGE imaging was performed $7-10 \mathrm{~min}$ after $0.15 \mathrm{mmol} / \mathrm{kg}$ of GBCA given as a bolus (Gadovist, Bayer Health Care) using phase sensitive inversion recovery. Analysis of volumes, mass, and function was performed off-line using Cvi42 software (Circle Cardiovascular Imaging, Calgary, Canada) according to the Society for CMR guidelines for reporting, with parameters indexed to height and body surface area (BSA), where appropriate [12]. Tissue tracking in the 3 long axis views was performed offline to assess $2 \mathrm{D}$ global longitudinal strain (GLS), according to previously published formulae [13]. Analysis of T1 was performed with manual contouring to define a region of interest (ROI) within the LV myocardium at basal and mid ventricular level (short-axis) on matched pre- and post-contrast images within the septum, as well as within the septum in the horizontal long axis view, and used to calculate myocardial extracellular volume (ECV) by validated formulae [14]. T1 values were measured in 'normal myocardium' defined by the absence of LGE. In addition, both short axis maps (basal and mid) were also manually contoured for endo- and epicardial borders, and partial voluming of blood minimised by using a $20 \%$ offset from the endoand epicardial border [15]. Stability of T1 measurement over time was confirmed by weekly analysis of a phantom within the magnet [16]. Analysis of all CMR parameters was performed by a single observer (SB) blinded to the identity of the subject, date, and time. A randomly selected subset of 10 patients were selected for intra- and inter-observer reproducibility studies by SB and RV. Bland-Altman plots were used to assess the reproducibility of these measurements, with mean differences calculated to identify any bias. Agreement was further quantified using intraclass correlation coefficients (ICCs), using two-way random effects models with absolute agreement.

\section{Phantom studies for T1 stability}

T1 times stability over time was assessed using a phantom made of high density plastic beads of agar/nickle chloride in plastic tubes. High-density polyethylene macrobeads encased these plastic tubes arranged in a $3 \times 3$ array, with resin layer at the base. Each tube represented a range of $\mathrm{T} 1$ times. These were scanned regularly for 12 months on a fortnightly basis using the T1 protocol described above. A tight shim was set with all images acquired at iso-center. The analysis was done using ROI as described above.

\section{Computed tomographic coronary angiography}

Participants without renal dysfunction had a single CT coronary artery calcium (CAC) score and CTCA performed on dual energy source 64 multi-slice scanner (Siemens Somaton Definition, Erlangen, Germany) using retrospective electrocardiogram (ECG) gated acquisition. A $20 \mathrm{~mL}$ test bolus of contrast was used to set delay and $80 \mathrm{~mL}$ of contrast given for final image acquisitions. Significant CAD was defined as a lesion $>70 \%$ on postmortem, invasive or CT coronary angiography.

\section{Statistical methods}

The baseline demographics of the cohort were summarised, with continuous variables reported as means \pm standard deviations (SDs) where normally distributed, and as medians and interquartile ranges (IQRs) otherwise. Changes over time in a range of markers were then assessed. Initially, Spearman's correlation coefficients (rho) were produced to measure the correlation between each marker and the timing of the measurement, relative to each patient's first scan. However, this approach did not account for the non-independence of the repeated scans on each patient. As a result, the analysis was repeated using a generalized estimating equation (GEE) approach. A separate model was produced for each marker, with the timing of the measurement, relative to each patient's first scan as a continuous covariate, and the patient identification number (ID) as a nominal factor. The patient ID was set as the subject effect, with the within-subject effect being the timing of the scan, relative to the initial scan, rounded to the nearest 6 months. 
An autoregressive (AR) (1) correlation structure was assumed throughout. Analyses were then performed to assess whether changes over time in T1 corresponded to changes in other markers. For each marker, separate linear regression models were produced for each patient that had data with at least two scans, with the timing of the measurement relative to baseline, as a covariate. The gradients from the resulting models were then used to represent the rate of change in the markers and were compared using Spearman's rho correlation coefficients. In addition, comparisons of gradients across dichotomous variables (e.g. gender) were performed using independent samples t-tests. All analyses were performed using IBM SPSS 22 (IBM Corp. Armonk, NY), with $\mathrm{p}<$ 0.05 deemed to be indicative of statistical significance throughout. For markers that were measured as percentages, gradients are reported as percentage point increases.

\section{Results}

\section{Study population}

There are 38 known adult ALMS patients within the United Kingdom, of whom 32 regularly attend the specialized service in Birmingham. Of these, two were excluded due to contra-indications to CMR (1 implantable cardioverter-defibrillator; 1 patient within research study), hence the analysis was based on the remaining 30. Baseline demographics and imaging data are presented in Table 1 and genetic data in Table 2. Family history of premature CAD was not recorded. The median age at the first scan was 21.3 years (IQR: 19.0-32.6), 63\% of patients were male and $67 \%$ were of White British ethnicity. The first CMR scan was performed the day before or same day as the first clinical attendance in the service. From first CMR, the median follow-up time was 45 months (maximum 67 months), during which time 76 additional scans were performed, a median of 3 follow-up scans per patient, with 4 patients only having a single scan during the follow-up period. More than half the cohort had conventional cardiovascular risk factors (Table 1). At baseline, LV volumes, ejection fraction (EF) and mass were within normal limits for age, sex and body surface area on CMR, although GLS was low-normal and six patients (20\%) had elevated NT-proBNP ( $\geq 144 \mathrm{ng} / \mathrm{L})$. In total, 14 patients had evidence of LGE. Of these, 2 patients had LGE at the insertion of the RV into the basal inferoseptum (insertion point LGE), 1 had focal subendocardial (mid anteroseptal LV segment) and 2 had focal epicardial (1 basal and mid inferolateral; 1 mid inferior and mid inferolateral LV segments) LGE. Mid wall LGE was present in 5 patients located within the basal and mid inferolateral LV segments, while 4 patients had extensive LV and RV LGE. The LGE pattern did not correspond to a specific coronary artery disease distribution.
Table 1 Baseline Patient Demographic and Cardiovascular Imaging Data

\begin{tabular}{|c|c|c|}
\hline Factor & $\mathbf{N}$ & Statistic \\
\hline \multicolumn{3}{|l|}{ Demographics } \\
\hline Age at first scan (years) & 30 & $21(19-33)$ \\
\hline Male Gender (\%) & 30 & 19 (63\%) \\
\hline White British (\%) & 30 & $20(67 \%)$ \\
\hline BMI $\left(\mathrm{kg} / \mathrm{m}^{2}\right)$ & 30 & $29(26-32)$ \\
\hline Systolic BP (mmHg) & 30 & $130 \pm 19$ \\
\hline Diastolic BP (mmHg) & 30 & $82 \pm 12$ \\
\hline Heart rate (beats/min) & 30 & $86 \pm 17$ \\
\hline \multicolumn{3}{|l|}{$C M R$} \\
\hline T1 Basal (ms) & 30 & 937 (913-1030) \\
\hline T1 SAX Basal ECV (\%) & 30 & $27(22-33)$ \\
\hline LVEDVi $\left(\mathrm{mL} / \mathrm{m}^{2}\right)$ & 30 & $58 \pm 12$ \\
\hline LVEF (\%) & 30 & $64 \pm 9$ \\
\hline LV mass index $\left(\mathrm{g} / \mathrm{m}^{2}\right)$ & 30 & $55(51-61)$ \\
\hline LAV index $\left(\mathrm{mL} / \mathrm{m}^{2}\right)$ & 30 & $34(25-43)$ \\
\hline GLS (\%) & 30 & $16 \pm 3$ \\
\hline \multicolumn{3}{|l|}{ Echocardiogram } \\
\hline E/A ratio & 29 & $1.5(1.3-1.6)$ \\
\hline Average E/e' ratio & 30 & $7.4 \pm 2.1$ \\
\hline $\mathrm{e}^{\prime}$ ave. $(\mathrm{cm} / \mathrm{s})$ & 27 & $9.1(7.7-11.0)$ \\
\hline \multicolumn{3}{|l|}{ Blood markers } \\
\hline Haemoglobin (g/dL) & 30 & $14.0 \pm 2.3$ \\
\hline $\mathrm{HbA1c}(\mathrm{mmol} / \mathrm{mol})$ & 29 & $49(39-71)$ \\
\hline C-peptide (pmol/L) & 30 & $3136(1516-4113)$ \\
\hline Cholesterol (mmol/L) & 30 & $4.8 \pm 1.3$ \\
\hline Triglycerides (mmol/L) & 30 & $2.5(1.7-4.2)$ \\
\hline NT-proBNP (ng/L) & 30 & $51(25-93)$ \\
\hline $\operatorname{eGFR}\left(\mathrm{mL} / \mathrm{min} / 1.73 \mathrm{~m}^{2}\right)$ & 30 & $108(62-123)$ \\
\hline \multicolumn{3}{|l|}{ Comorbidities } \\
\hline Hypertension & 30 & $17(57 \%)$ \\
\hline Dyslipidaemia & 30 & $18(60 \%)$ \\
\hline Infantile cardiomyopathy & 30 & $12(40 \%)$ \\
\hline Diabetes & 30 & $18(60 \%)$ \\
\hline CKD & 30 & $18(60 \%)$ \\
\hline
\end{tabular}

$B M I$ indicates body mass index, $B P$ blood pressure, $C M R$ cardiovascular magnetic resonance, $E / A$ ratio mitral early filling $(E) /$ atrial filling $(A)$ ratio, $E / e^{\prime}$ mitral early filling (E)/early myocardial relaxation velocity (e'), ECV extracellular volume, eGFR estimated glomerular filtration rate, GLS global longitudinal strain, HbA1c glycated haemoglobin A1c, IQR interquartile range, $L A V$ left atrial volume, $L V$ left ventricular, $L V E D V i$ left ventricular end-diastolic volume indexed to body surface area, LVEF left ventricular ejection fraction, LVESVi left ventricular end-systolic volume indexed, NT-proBNP serum Nterminal pro $B$ natriuretic peptide, $R V$ right ventricle, $S A X$ short axis, $C K D$ chronic kidney disease

Data are reported as $\mathrm{N}(\%)$, mean $\pm \mathrm{SD}$, or median (IQR), as applicable 
Table 2 Mutations in ALMS1 gene in study participants

\begin{tabular}{|c|c|c|c|c|c|c|c|c|}
\hline Mutation 1 & Exome 1 & $\begin{array}{l}\text { Allele } 1 \\
\text { nucleotide } \\
\text { change }\end{array}$ & $\begin{array}{l}\text { Allele_1 amino } \\
\text { acid change }\end{array}$ & $\begin{array}{l}\text { Homo/ } \\
\text { Hetero/CH }\end{array}$ & Mutation 2 & Exome 2 & $\begin{array}{l}\text { Allele } 2 \\
\text { nucleotide } \\
\text { change }\end{array}$ & $\begin{array}{l}\text { Allele_2 amino } \\
\text { acid change }\end{array}$ \\
\hline Nonsense & 8 & c. $2041 C>T$ & p.Arg681*X & Homo & ?Nonsense & 8 & c. $2041 C>T$ & p.Arg681* \\
\hline Nonsense & 8 & c. $6823 \mathrm{C}>\mathrm{T}$ & p.Arg2275* & $\mathrm{CH}$ & Nonsense & 10 & c. $9535 \mathrm{C}>\mathrm{T}$ & p.Arg3179* \\
\hline Nonsense & 8 & c. $2822 \mathrm{~T}>\mathrm{A}$ & p.Leu941* & $\mathrm{CH}$ & Frameshift & 16 & c.10775delC & p.Thr3592Lysfs*6 \\
\hline Frameshift & 8 & c.6584delA & p.Lys2195Serf*10 & $\mathrm{CH}$ & Nonsense & 5 & c.1008_1009delTG & p.Cys336fs*1 \\
\hline Nonsense & 10 & c. $8002 C>T$ & p.Arg2668* & $\mathrm{CH}$ & Nonsense & 16 & c. $10879 C>T$ & pArg3627* \\
\hline Nonsense & 10 & c. $9001 \mathrm{C}>\mathrm{T}$ & p.Gln3001* & Hetero & & & & \\
\hline Frameshift & 8 & c.6895delG & p.val2299Trps*43 & $\mathrm{CH}$ & Frameshift & 16 & c. $11443 C>T$ & p.Gln3815* \\
\hline Nonsense & 16 & $11107 \mathrm{C}>\mathrm{T}$ & p.Arg3703* & Hetero & & & & \\
\hline Nonsense & 16 & $11107 \mathrm{C}>\mathrm{T}$ & p.Arg3703* & Homo & Nonsense & 16 & $11107 C>T$ & p.Arg3703* \\
\hline Frameshift & 16 & c.10579_1580delAT & p.Met3527Valfs*13 & $\mathrm{CH}$ & Frameshift & 18 & c.11856delC & p.Asn3952Lysfs*41 \\
\hline Frameshift & 16 & c.10769delC & p.Thr3590Lysfs*6 & $\mathrm{CH}$ & Missense & 8 & c. $5356 \mathrm{~A}>\mathrm{G}$ & p.Asn1786Asp \\
\hline Nonsense & 16 & c. $11107 C>T$ & p.Arg3703* & Hetero & & & & \\
\hline Nonsense & 8 & c. $6823 \mathrm{C}>\mathrm{T}$ & p.Arg2275* & $\mathrm{CH}$ & Nonsense & 10 & c. $9535 \mathrm{C}>\mathrm{T}$ & p.Arg3179* \\
\hline \multirow[t]{2}{*}{ Frameshift } & 8 & c.1729delA & p.Arg577Glyfs*17 & $\mathrm{CH}$ & Nonsense & 16 & c. $10477 C>T$ & p.Gln3493* \\
\hline & 8 & $c 6526 C>T$ & p.G $\ln 217^{*}$ & Hetero & & & & \\
\hline Nonsense & 10 & c.8932C > T & p.Gln2978* & $\mathrm{CH}$ & Missense & 8 & c. $5356 \mathrm{~A}>\mathrm{G}$ & p.Asn1786Asp \\
\hline Nonsense & 8 & c. 4937 C > A & p.Ser1646* & Homo & Nonsense & 8 & c. 4937 C > A & p.Ser1646* \\
\hline \multirow[t]{2}{*}{ Nonsense } & 8 & c.4937C > A & p.Ser1646* & Homo & Nonsense & 8 & c.4937C > A & p.Ser1646* \\
\hline & 9 & c.7544- & & Homo & Exon & 9 & c.7544- & \\
\hline Deletion & & 200_7677+1110del & & & deletion & & 200_7677+1110del & \\
\hline Nonsense & 8 & c. 4937 C > A & p.Ser2646* & Hetero & Nonsense & 8 & c.6526C > T & p.Gln2176* \\
\hline Nonsense & 8 & c. 6299 C > A & p.Ser2100* & $\mathrm{CH}$ & Nonsense & 16 & c. $10477 C>$ T & p.Gln3493* \\
\hline Nonsense & 8 & c. 6299 C > A & p.Ser2100* & $\mathrm{CH}$ & Nonsense & 16 & c. $10477 C>$ T & p.Gln3493* \\
\hline Frameshift & 16 & c.10769delC & p.Thr3590Lysfs*6 & $\mathrm{CH}$ & Missense & 16 & c. $11410 C>T$ & p.Arg38404* \\
\hline Exon & 9 & c.7544- & & Homo & Exon & 9 & c.7544- & \\
\hline deletion & & 200_7677+1110del & & & deletion & & 200_7677+1110del & \\
\hline Nonsense & 8 & c. $2041 C>T$ & p.Arg681* & Homo & Nonsense & 8 & c. $2041 C>T$ & p.Arg681* \\
\hline Nonsense & 8 & c. $2041 C>T$ & p.Arg681* & Homo & ?Nonsense & 8 & c. $2041 C>T$ & p.Arg681* \\
\hline Exon deletion & 9 & c.7544- & & Homo & Exon deletion & 9 & $\begin{array}{l}\text { c.7544-200_7677 } \\
+1110 \text { del }\end{array}$ & \\
\hline Nonsense & 16 & 200_7677+1110del & p.Gln3495* & $\mathrm{CH}$ & Frameshift & 16 & c.10775delC & p.Thr3592Lysfs*6 \\
\hline Nonsense & 16 & $11107 C>T$ & p.Arg3703* & Hetero & & & & \\
\hline Frameshift & 10 & c.7911dupC & p.Asn2638Glnfs*24 & Homo & Frameshift & 10 & c.7911dupC & p.Asn2638Glnfs*24 \\
\hline
\end{tabular}

$\mathrm{CH}$ indicates compound heterozygote, Hetero, Heterozygote; Homo, homozygote

\section{Serial data}

On correlation analysis, T1 basal and mid ventricular level (short-axis; both $\mathrm{p}<0.001$ ), ECV basal and mid ventricular level (short-axis; $p=0.081$ and $\mathrm{p}=0.064$ ), and LV mass $(p=0.008)$ all increased over time. Analysis of serial measurements using a GEE approach found significant increases over time in T1, as measured on the basal and mid ventricular (short-axis) levels, with similar gradients of $25.8 \mathrm{~ms}$ per year (95\% CI: 20.0-31.7, $p<0.001$ ) and 21.8 ms per year (95\% CI: 17.4-26.1, $p<0.001$ ), respectively
(Fig. 1a). Significant increases in ECV were also observed, with gradients of 1.4 percentage points per year (95\% CI: $0.8-2.0, p<0.001)$ and 1.1 percentage points per year (95\% CI: $0.6-1.6, p<0.001$ ) for basal and mid ventricular levels, respectively (Fig. 1b). Figure 2 shows an example of changes in $\mathrm{T} 1$ for a specific individual. In addition, the LV mass index was found to increase significantly over time, by an average of $2.8 \mathrm{~g} / \mathrm{m}^{2}$ per year $\left(95 \% \mathrm{CI}: 1.9-3.7 \mathrm{~g} / \mathrm{m}^{2}\right.$, $p<0.001)$. Analyses using a correlation approach returned consistent findings (Table 3). 


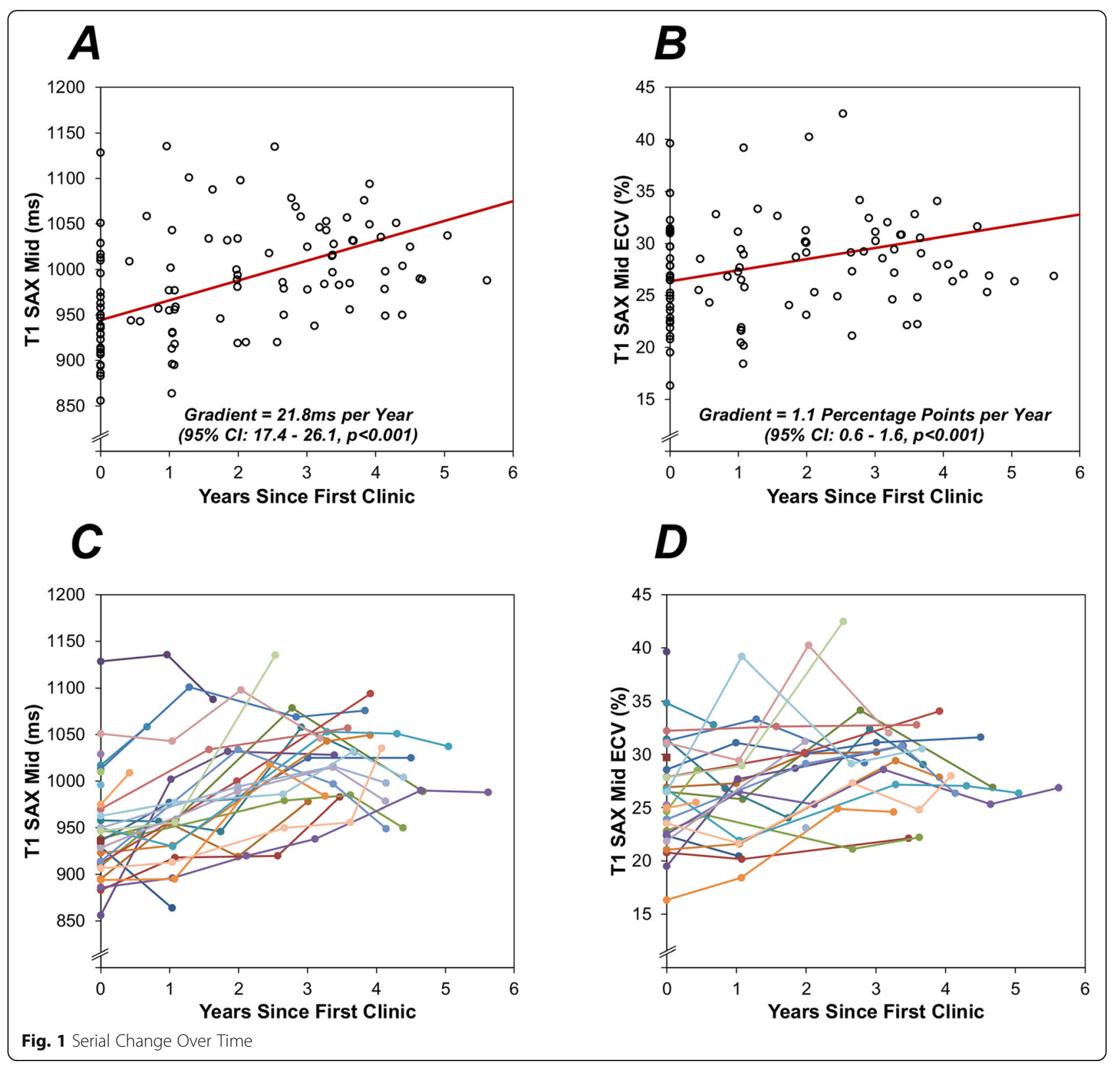

Two patients developed LGE during follow up, 1 of whom developed new RV insertion point LGE and 1 developed subendocardial LGE in the mid anteroseptum (participants 10 \& 11). No significant changes over time were detected in LV end-diastolic volume indexed to BSA (LVEDVi, $p=0.909$ ), LV end-systolic volume indexed to BSA (LVESVi, $p=0.067$ ), LV ejection fraction (EF, $p=0.575)$, left atrial volume indexed (LAVi, $p=0.409$ ), or LV GLS (GLS, $p=0.809$ ). In addition, no significant changes over time were noted in diastolic function on echocardiography, including mitral early filling $(\mathrm{E})$ /atrial filling $(\mathrm{A})$ ratio $(\mathrm{p}=0.313)$, and average E/early myocardial relaxation velocity (e', $p=0.299)$ (Table 3).
Factors associated with change in ECV or T1

Changes in ECV and T1 were not found to be significantly associated with differences in LV volumes, mass, or EF over follow-up (Table 4). Although there was a significant association between increasing $\mathrm{T} 1$ and impaired (less negative) GLS (Rho 0.422, $p=0.036$, this relationship was not mirrored by increasing ECV. Moreover, there was also no evidence of a significant association between the change in ECV or T1 and markers of diastolic function (E/A; E/e' average; LAV). There was no evidence of a significant relationship between change in ECV or T1 with age, hypertension, diabetes, history of infantile cardiomyopathy, or presence/absence of coronary artery disease (CAD) (Table 5). 
A. Mid SAX T1 map at baseline (patient 1) B. Mid SAX T1 map at 4 years (patient 1)

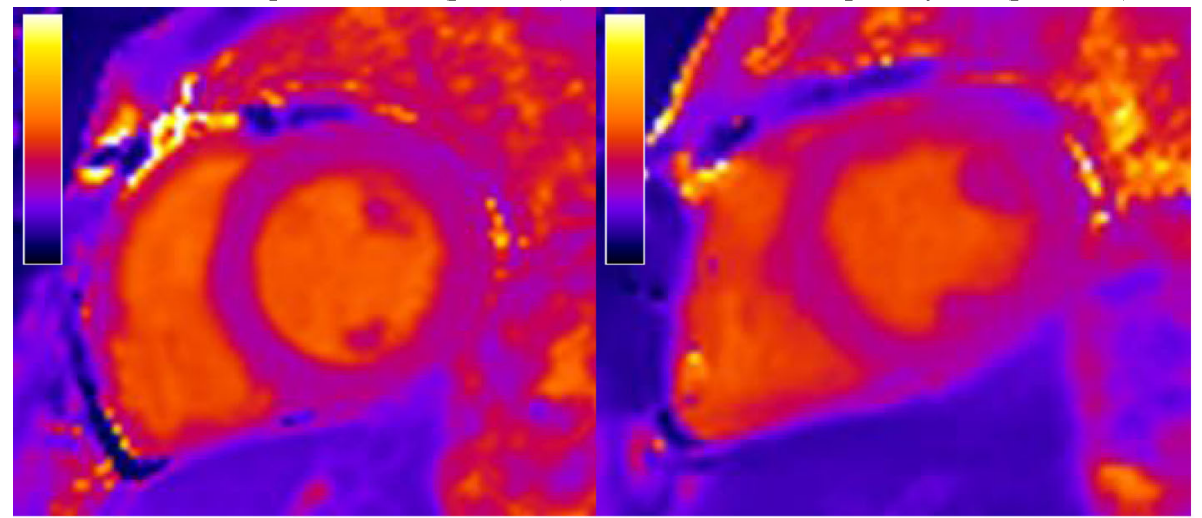

C. Modified SAX T1 map at baseline

D. Modified SAX T1 map at 4 years

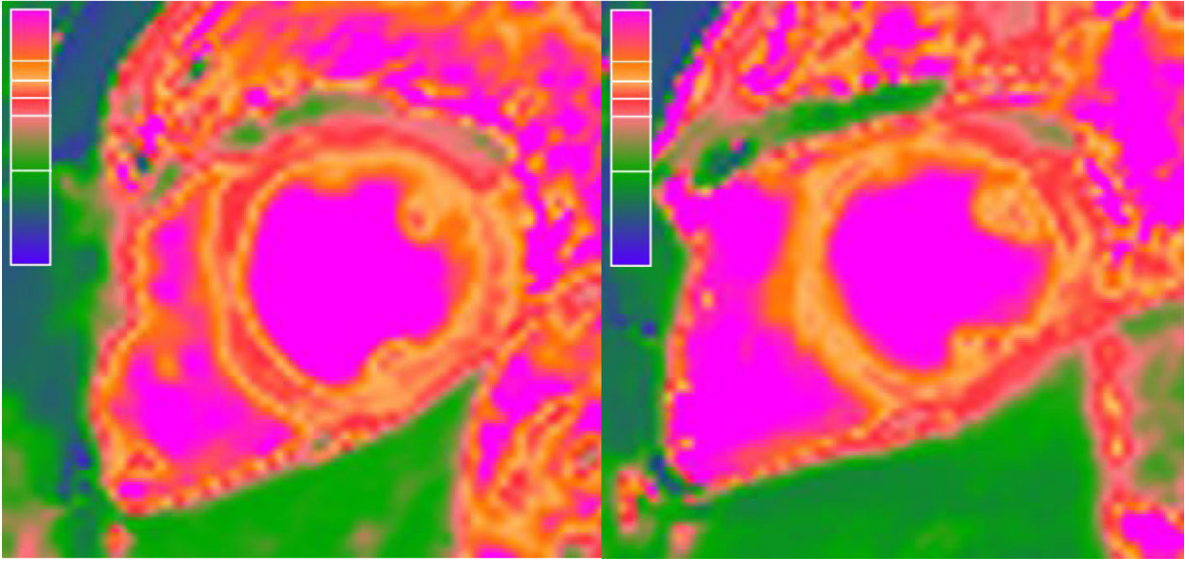

Fig. 2 Changes in T1 Mapping Over Time Within an Individual. a Mid SAX T1 map at baseline (patient 1) b Mid SAX T1 map at 4 years (patient 1). c Modified SAX T1 map at baseline. d Modified SAX T1 map at 4 years. a represents T1 maps at baseline (T1 in ROI 923 ms) and $\mathbf{b}$ represent the increase T1 times at 4 years (T1 in ROI $1012 \mathrm{~ms}$ ). The modified T1 maps (c \& $\mathbf{d})$ of the original maps $(\mathbf{a} \& \mathbf{b})$ have been colour coded to show the difference. The scale represents an increase in T1 toward the top. SAX, short axis slice

\section{Coronary artery disease}

Presence or absence of CAD could not be established in 3 of the 30 patients. One patient was not assessed, as they refused to undergo CAC/CTCA due to a combination of claustrophobia and needle phobia, while 2 patients had not undergone CAD assessment at time of data collection. Of the remaining 27 patients, 3 patients presented with symptoms and were diagnosed with CAD. Of these, one died before a CTCA could be performed, but post-mortem showed a right coronary artery occluded with thrombus. The second patient had invasive coronary angiography following onset of symptoms and was found to have severe CAD. The third patient has $\operatorname{CAD}(>70 \%$ stenosis in the mid left anterior descending artery) identified on CTCA and was treated by percutaneous coronary intervention with stent implantation. The remaining 24 asymptomatic patients all had a CTCA done, and 22 had CAC scoring. Of these, 4 were found to have elevated CAC Agatston scores of 48, 69, 156, and 209. These 4 patients were found to have mild atheroma with no flow-limiting stenosis, with CTCA being normal in the remainder. In summary, a total of 3/ 27 (11\%) had significant CAD defined by $>70 \%$ stenosis, whilst a further $4 / 27$ (15\%) had non flow-limiting atheroma. Further details about these 7 patients are reported in Table 6.

\section{Intra- and inter-observer reproducibility, phantom study and Normal ranges}

Ten CMR studies were randomly selected for assessment of intra- and inter-observer variation. There was no consistent pattern of inter-observer bias on Bland-Altman analyses. Intra-observer mean bias for basal T1 was 5.8 ms (95\% CI -4.3, $15.8 \mathrm{~ms})$, with an ICC (random model) of $0.9(p<0.001)$, whilst inter-observer mean bias for basal T1 was $6.4 \mathrm{~ms}(95 \% \mathrm{CI}-7.2,20 \mathrm{~ms})$, with an ICC of $(0.9, p<0.001)$. Normal T1 measurements and ECV values using the same protocol in normal volunteers on the same scanner were as follows: $(n=26)$ mid short axis (SAX) pre contrast T1 $970 \pm 11 \mathrm{~ms}$; post contrast T1 
Table 3 Changes over time in markers

\begin{tabular}{|c|c|c|c|c|c|c|}
\hline & No. & No. & Correla & & GEE Analysis & \\
\hline & Patients & Scans & Rho & $p$-Value & Gradient (95\% Cl) & $p$-Value \\
\hline T1 SAX Basal (ms) & 30 & 104 & 0.415 & $<0.001$ & $25.8(20.0,31.7)$ & $<0.001$ \\
\hline T1 SAX Mid (ms) & 30 & 104 & 0.451 & $<0.001$ & $21.8(17.4,26.1)$ & $<0.001$ \\
\hline T1 SAX Basal ECV (\%, pp) & 30 & 94 & 0.181 & 0.081 & $1.4(0.8,2)$ & $<0.001$ \\
\hline T1 SAX Mid ECV $(\%, p p)$ & 30 & 94 & 0.192 & 0.064 & $1.1(0.6,1.6)$ & $<0.001$ \\
\hline LVEDV Index $\left(\mathrm{ml} / \mathrm{m}^{2}\right)$ & 30 & 106 & -0.113 & 0.247 & $0.04(-0.60,0.68)$ & 0.909 \\
\hline LVESV Index (m1/m2) & 30 & 106 & -0.164 & 0.094 & $0.92(-0.06,1.90)$ & 0.067 \\
\hline $\operatorname{LVEF}(\%, \mathrm{pp})$ & 30 & 106 & 0.104 & 0.289 & $-0.27(-1.23,0.68)$ & 0.575 \\
\hline LV Mass Index $\left(\mathrm{g} / \mathrm{m}^{2}\right)$ & 30 & 104 & 0.257 & 0.008 & $2.8(1.9,3.7)$ & $<0.001$ \\
\hline LAV Biplane $\left(\mathrm{ml} / \mathrm{m}^{2}\right)$ & 29 & 60 & -0.221 & 0.090 & $-2.0(-6.7,2.7)$ & 0.409 \\
\hline GLS & 30 & 95 & 0.032 & 0.756 & $-0.04(-0.33,0.26)$ & 0.809 \\
\hline GRS & 28 & 86 & 0.010 & 0.924 & $-0.36(-1.18,0.47)$ & 0.394 \\
\hline $\mathrm{E} / \mathrm{A}$ & 29 & 72 & 0.133 & 0.267 & $2.3(-2.2,6.9)$ & 0.313 \\
\hline E/e' Average & 30 & 68 & 0.161 & 0.189 & $0.3(-0.2,0.8)$ & 0.299 \\
\hline HBA1c (mmol/mol) & 29 & 100 & -0.116 & 0.250 & $-0.9(-2.5,0.7)$ & 0.284 \\
\hline C-Peptide (pmol/L) & 30 & 94 & -0.028 & 0.788 & $-90.7(-364.6,183.2)$ & 0.516 \\
\hline NTpro BNP (ng/L) & 30 & 105 & -0.101 & 0.306 & $34.4(-33.9,102.8)$ & 0.324 \\
\hline
\end{tabular}

Correlation analysis - results are from Spearman's rho correlation coefficients between the marker and the timing of the scan, relative to the baseline scan GEE analysis - results are from generalised estimating equation models, as described in the methods. The "gradient" represents the average yearly change in the marker over the period

Bold $p$-values are significant at $p<0.05$. ECV and EF were measured as percentages, hence gradients represent percentage point (pp) increases

$E / A$ mitral early filling (E)/atrial filling (A), E/e', mitral early filling (E)/early myocardial relaxation velocity (e'), ECV extracellular volume, HbA1c glycated haemoglobin A1c, LAV left atrial volume, LV left ventricular, LVEDV index, left ventricular end-diastolic volume indexed to body surface area, $L V E F$ left ventricular ejection fraction, LVESV index, left ventricular end-systolic volume indexed, NT-proBNP serum Nterminal pro B natriuretic peptide; SAX, short axis

Table 4 Associations Between T1/ECV Over Time and Changes in Myocardial Structure and Function

\begin{tabular}{|c|c|c|c|c|c|c|}
\hline \multirow[t]{2}{*}{ Gradient in } & \multicolumn{3}{|c|}{$\begin{array}{l}\text { T1 SAX Mid } \\
\text { Gradient }\end{array}$} & \multicolumn{3}{|c|}{$\begin{array}{l}\text { T1 SAX Mid ECV } \\
\text { Gradient }\end{array}$} \\
\hline & $N$ & Rho & p-Value & $N$ & Rho & p-Value \\
\hline LVEDV Index $\left(\mathrm{ml} / \mathrm{m}^{2}\right)$ & 26 & 0.019 & 0.927 & 24 & -0.052 & 0.809 \\
\hline LVESV Index $\left(\mathrm{ml} / \mathrm{m}^{2}\right)$ & 26 & -0.444 & 0.023 & 24 & -0.188 & 0.379 \\
\hline LVEF (\%) & 24 & 0.352 & 0.091 & 22 & 0.088 & 0.699 \\
\hline LV Mass Index $\left(\mathrm{g} / \mathrm{m}^{2}\right)$ & 26 & -0.056 & 0.784 & 24 & 0.203 & 0.340 \\
\hline LAV Biplane $\left(\mathrm{ml} / \mathrm{m}^{2}\right)$ & 20 & 0.245 & 0.298 & 18 & -0.377 & 0.123 \\
\hline GLS & 25 & 0.422 & 0.036 & 23 & 0.024 & 0.914 \\
\hline GRS & 23 & 0.268 & 0.217 & 21 & 0.116 & 0.618 \\
\hline$E / A$ & 22 & -0.292 & 0.187 & 20 & -0.029 & 0.905 \\
\hline E/e' Average & 23 & -0.370 & 0.083 & 21 & -0.268 & 0.241 \\
\hline HBA1c (mmol/mol) & 24 & -0.119 & 0.579 & 22 & 0.189 & 0.399 \\
\hline C-Peptide (pmol/L) & 26 & -0.101 & 0.624 & 24 & -0.037 & 0.865 \\
\hline Pro NT BNP (ng/L) & 26 & -0.072 & 0.726 & 24 & 0.023 & 0.913 \\
\hline
\end{tabular}

For each of the markers considered, a linear regression model was produced for each patient, with the timing of the scan, relative to the first scan, set as a continuous covariate. Only those patients with at least two valid scans for the stated marker were included in the analysis. Spearman's (rho) correlation coefficients were then produced between the resulting gradients. Bold $\mathrm{p}$ values are significant at $p<0.05$

$E / A$ mitral early filling (E)/atrial filling (A), E/e', mitral early filling (E)/early myocardial relaxation velocity (e'), ECV extracellular volume, $H b A 1 c$ glycated haemoglobin A1c, LAV left atrial volume, LV left ventricular, LVEDV index left ventricular end-diastolic volume indexed to body surface area, LVEF, left ventricular ejection fraction; LVESV index, left ventricular end-systolic volume indexed; NT-proBNP, serum Nterminal pro B natriuretic peptide; SAX, short axis
$522 \pm 58 \mathrm{~ms} ;$ ECV $25 \pm 1 \%$. Over 12 months, 25 MOLLI datasets were collected. The scanner room temperature was stable at $20.96 \pm 0.98 \mathrm{C}$. Coefficients of variation across the 9 tubes were stable and ranged from 0.436 to $0.872 \%$ when unadjusted for temperature and improved to and 0.436 to $0.868 \%$ with correction for room temperature.

\section{Discussion}

This is the first prospective study to demonstrate an increase in $\mathrm{T} 1$ and ECV over time, paralleled by an increase in LV mass in ALMS. Despite changes in T1 and $\mathrm{ECV}$, there was no association with serial changes in systolic or diastolic contractile dysfunction, either in terms of EF, E/A, E/e', LA volume, or elevation in biomarker (NT-proBNP). Although increasing native T1 over time was associated with impaired (less negative) GLS, this was an isolated relationship that was not mirrored in relation to ECV and was not associated with other changes in functional parameters, The increased T1 and ECV were not significantly associated with the presence or absence of CAD. These data are consistent with mild, progressive myocardial fibrosis over time in ALMS, although no structural or consistent functional consequences were noted within a median three and a half years' follow up. Why is this finding of importance? ALMS is characterized by obesity, insulin resistance and dyslipidaemia, diseases that are common but are 
Table 5 Association between baseline factors and changes in T1/ECV

\begin{tabular}{|c|c|c|c|c|c|c|}
\hline & T1 S & Gradient (m & & T1 & CV Gradient ( & ar) \\
\hline & $N$ & Mean $\pm S D$ & p-Value & $N$ & Mean $\pm S D$ & $p$-Value \\
\hline Age at First Scan* & 26 & $-0.137^{*}$ & $0.505^{*}$ & 24 & 0.169 & 0.430 \\
\hline Ethnicity & & & 0.278 & & & 0.360 \\
\hline White British & 18 & $26.3 \pm 25.2$ & & 17 & $1.8 \pm 2.5$ & \\
\hline Other & 8 & $12.7 \pm 35.9$ & & 7 & $0.7 \pm 2.6$ & \\
\hline Gender & & & 0.112 & & & 0.864 \\
\hline Male & 17 & $15.5 \pm 29.4$ & & 15 & $1.4 \pm 2.5$ & \\
\hline Female & 9 & $34.5 \pm 24.8$ & & 9 & $1.6 \pm 2.8$ & \\
\hline ACE/ARB & & & 0.969 & & & 0.771 \\
\hline No & 6 & $21.7 \pm 56.6$ & & 5 & $1.8 \pm 2.9$ & \\
\hline Yes & 20 & $22.2 \pm 16.0$ & & 19 & $1.4 \pm 2.5$ & \\
\hline Statins/Fibrates & & & 0.103 & & & 0.121 \\
\hline No & 14 & $30.7 \pm 22.5$ & & 14 & $2.1 \pm 2.8$ & \\
\hline Yes & 12 & $12.1 \pm 33.1$ & & 10 & $0.5 \pm 1.7$ & \\
\hline Hypertension & & & 0.930 & & & 0.117 \\
\hline No & 11 & $21.5 \pm 35.6$ & & 10 & $0.5 \pm 2.4$ & \\
\hline Yes & 15 & $22.5 \pm 24.2$ & & 14 & $2.1 \pm 2.4$ & \\
\hline Hyperlipidaemia & & & 0.040 & & & 0.989 \\
\hline No & 11 & $35.5 \pm 21.8$ & & 11 & $1.5 \pm 2.2$ & \\
\hline Yes & 15 & $12.3 \pm 30.1$ & & 13 & $1.5 \pm 2.8$ & \\
\hline Infantile cardiomyopathy & & & 0.710 & & & 0.904 \\
\hline No & 16 & $20.4 \pm 33.0$ & & 15 & $1.5 \pm 2.9$ & \\
\hline Yes & 10 & $24.9 \pm 22.0$ & & 9 & $1.4 \pm 1.9$ & \\
\hline Diabetes & & & 0.764 & & & 0.132 \\
\hline No & 9 & $19.7 \pm 39.3$ & & 9 & $0.5 \pm 2.5$ & \\
\hline Yes & 17 & $23.4 \pm 23.0$ & & 15 & $2.1 \pm 2.4$ & \\
\hline Insulin resistance (not diabetes) & & & 0.752 & & & 0.870 \\
\hline No & 20 & $21.1 \pm 30.2$ & & 18 & $1.5 \pm 2.6$ & \\
\hline Yes & 6 & $25.5 \pm 26.3$ & & 6 & $1.3 \pm 2.4$ & \\
\hline CKD & & & 0.889 & & & 0.630 \\
\hline No & 12 & $23.0 \pm 33.3$ & & 12 & $1.2 \pm 2.0$ & \\
\hline Yes & 14 & $21.4 \pm 25.8$ & & 12 & $1.7 \pm 3.0$ & \\
\hline Presence of CAD** & & & 0.622 & & & 0.121 \\
\hline No & 20 & $23.5 \pm 27.5$ & & 20 & $1.0 \pm 2.0$ & \\
\hline Yes & 3 & $32.7 \pm 44.7$ & & 3 & $3.4 \pm 4.7$ & \\
\hline
\end{tabular}

Gradients were calculated on a per-patient basis, as described in the methods, with the resulting values reported as mean \pm SD, and compared between groups using independent samples t-tests, unless stated otherwise. Only those patients with at least two valid scans for the stated marker were included in the analysis *Reported as a Spearman's rho correlation coefficient and $p$-value. ${ }^{*}$ Excludes $N=3$ patients with unknown CAD status. Note that ECV was measured as a percentage, hence gradients are reported in percentage points ( $\mathrm{pp}$ ) per year. Bold $\mathrm{p}$-values are significant at $p<0.05$

projected to increase beyond that expected by evolving population demographics [17]. Furthermore, heart failure is highly prevalent in each, with adverse prognosis whether or not associated with coronary artery disease [18]. Given the premature onset of cardiomyopathy, ALMS is a paradigm to investigate the role of DIF in pathogenesis of heart failure, and whether this may be a target for therapeutic intervention [19] or simply a bystander to other disease processes [20]. In such a population, particularly one with a high rate of hypertension at baseline, it is of interest that while indexed LV mass increased, there was no relationship with change in T1 or ECV. Our data identifies progression of DIF that is independent of coronary artery disease, but further 
Table 6 Details of Seven Patients with Coronary Artery Disease or Coronary Artery Atheroma

\begin{tabular}{|c|c|c|c|c|c|c|c|}
\hline Patient ID & 1 & 2 & 3 & 4 & 5 & 6 & 7 \\
\hline Age & 38 & 42 & 19 & 48 & 36 & 43 & 19 \\
\hline Gender & M & M & M & M & M & $\mathrm{F}$ & M \\
\hline Agatston & PM & ICA & 1 & 48 & 69 & 156 & 209 \\
\hline Findings & RCA Occlusion & $P C I$ to $L A D$ & $\begin{array}{l}\text { Mild } \\
\text { atheroma }\end{array}$ & Mild atheroma & $\begin{array}{l}\text { Mild } \\
\text { atheroma }\end{array}$ & Mild atheroma & LAD stenosis \\
\hline LGE Pattern & Extensive diffuse & Focal epicardial & $\begin{array}{l}\text { Mid wall } \\
\text { LGE }\end{array}$ & Extensive diffuse & $\begin{array}{l}\text { Mid wall } \\
\text { LGE }\end{array}$ & Extensive diffuse & $\begin{array}{l}\text { Focal } \\
\text { epicardial }\end{array}$ \\
\hline LGE territory & $\begin{array}{l}\text { Basal infero-lateral } \\
\text { segment, basal, mid } \\
\text { and apical lateral and } \\
\text { inferior RV }\end{array}$ & $\begin{array}{l}\text { Basal inferolateral and } \\
\text { mid inferolateral } \\
\text { walls. }\end{array}$ & $\begin{array}{l}\text { Basal infero- } \\
\text { lateral } \\
\text { segment }\end{array}$ & $\begin{array}{l}\text { Basal inferolateral } \\
\text { and mid inferolateral } \\
\text { transmural in the mid } \\
\text { and apical anterior }\end{array}$ & $\begin{array}{l}\text { Basal } \\
\text { inferolateral }\end{array}$ & $\begin{array}{l}\text { Basal and mid LV } \\
\text { levels, mid antero- } \\
\text { lateral, mid infero- } \\
\text { lateral, mid inferior } \\
\text { segments }\end{array}$ & $\begin{array}{l}\text { Mid inferior } \\
\text { and mid } \\
\text { infero-lateral } \\
\text { LV segments. }\end{array}$ \\
\hline HTN & Y & Y & Y & Y & Y & Y & N \\
\hline Hyperlipidaemia & Y & Y & Y & Y & Y & Y & Y \\
\hline Diabetes & Y & Y & $\mathbb{R}$ & Y & N & $\mathrm{N}$ & Y \\
\hline CKD Stage & 2 & 5 & 0 & 2 & 1 & 1 & 2 \\
\hline Infantile CM & N & Y & N & $N$ & Y & Y & Y \\
\hline
\end{tabular}

ICA invasive coronary angiography, CKD chronic kidney disease stage, CM cardiomyopathy, HTN hypertension, ID identification number, LAD left anterior descending, $P M$ Post mortem finding, $N$ no, $Y$ yes

research is needed to clarify relationship with functional decline.

Previous studies have demonstrated that $\mathrm{T} 1$ values and ECV are increased in ALMS compared to control subjects $[3,4]$. These data are consistent with postmortem histology studies demonstrating both coarse and DIF in adults with ALMS $[1,21,22]$. Although the data correlating high T1, increased ECV, and histology in ALMS are limited [4], T1 mapping and ECV on CMR as markers of DIF have been validated histologically in other conditions [23-25]. Longitudinal studies have shown T1 mapping to be a robust parameter, with little variability in healthy individuals over time, and in our study, the increase was measured in a single magnet using the same sequence with stable signal confirmed by phantom [26]. The increase in T1 and ECV was not, however, associated with a change in systolic or diastolic contractility, other than an isolated association between native T1 and GLS, measured either by CMR or echocardiography. In our previous cross-sectional study, an association was found between increased T1 and ECV and altered GLS measured using CMR tagging, and it may be that the method used in this serial study, tissuetracking, was less sensitive [4]. It is also notable that in our previous and current work, a significant proportion of the ALMS population have both native T1 and ECV within normal range. In fact, baseline values in the current study for both native T1 and ECV were below the reference values for normal subjects, an effect which is usually attributable either to deposition of lipid or accumulation of iron, neither of which have been described in the limited histological data in adults with
ALMS. Furthermore, the size of the change was small and it is possible either that change in function may require longer follow-up to have a significant impact, or that progression of fibrosis may be variable between individuals. This is consistent with lack of functional change on serial CMR over 2 years seen in patients with chronic stable cardiomyopathy who had a detectable increase in T1 times [27]. Given the potential signal of an association between increasing native $\mathrm{T} 1$ and impaired GLS, further prospective studies are needed to see if the increased T1 and ECV are associated in the longer term with the development of systolic dysfunction, heart failure and death, as has been demonstrated in other populations [28-31].

Infantile cardiomyopathy is a common finding in infants with ALMS, presenting within the first 3 months of life [32]. The majority (74\%) survive, with response to standard heart failure therapy resulting in recovery of cardiac function in the majority [2]. Recent evidence suggests that the ALMS1 protein has a role in perinatal cardiomyocyte cell division and replication, and that its deficiency can cause mitogenic cardiomyopathy [33, 34]. The mechanisms leading to infantile cardiomyopathy appear to differ from those seen in adult cardiomyopathy, where myocardial fibrosis plays a dominant role. In keeping with this, there was no detectable difference in structure, function, or progression of T1/ECV between those patients with and those without a history of infantile disease. In addition, given the risk profile of the ALMS cohort under care, it was thought important to investigate the potential impact of CAD on the development of myocardial fibrosis and heart failure. More than 
a quarter of the cohort (7/27) had evidence of coronary atheroma, although only 3 patients had flow-limiting disease, 2 of whom were symptomatic. The prevalence of $\mathrm{CAD}$ was not found to influence the progression of $\mathrm{T} 1$ or ECV significantly in this study, although the statistical power of this analysis was low due to small numbers.

Limitations. The primary limitation of this study, inevitably, is the relatively small sample size. As a result of this, the statistical power of analyses is low, meaning that only relatively large effects would have been detecteable, increasing the risk of false negatives. This was especially applicable to the analyses comparing the $\mathrm{T} 1$ and ECV gradients across baseline factors, particularly for comparisons across groups with unequal numbers of patients, such as the presence of CAD ( $N=3$ vs. $N=20$ ). Although numbers were small, participants had a genetically confirmed rare disease, recruited from a national centre with one of the largest adult cohorts available. Where significant trends over time were detected, the rates of change were generally small, and may not have been sufficient to have clinical impact in the shortterm. However, the fact that the trajectories were similar across the whole cohort, and that testing of the scanner using a phantom within the magnet used demonstrated marked stability of the signal over time, would suggest that these trends are reflective of genuine progression of fibrosis. Further research is needed in a larger cohort, which is likely to require organisation of an international registry.

Baseline T1 values were heterogeneous in our cohort, consistent with our previous and other research in ALMS [3], although this may in part be accounted for differences in age, gender and co-existing metabolic status. This significant inter-individual variation suggests that development of fibrosis is not an inevitable consequence of the genetic defect alone and that environmental factors may also play an important role. We acknowledge however, the possibility of myocardial fat deposition or pseudo normalization of $\mathrm{T} 1$ due to the combined presence of lipid and fibrosis within the LV. Our own limited post-mortem data did not identify significant myocardial fat deposition in ALMS but recognise that further tissue characterisation, including T2 mapping, would have been enlightening. It is also important to note that distribution of mid wall LGE has a predilection for the inferolateral wall, which is seen in a number of different diseases, but is not limited to that location, as in early male Fabry disease, which is consistent with our hypothesis that fibrosis is not inevitable but may be subject to individual and environmental factors.

\section{Conclusion}

This is the first comprehensive longitudinal study to suggest that myocardial fibrosis in ALMS progresses over time. There were no associated functional changes in systolic or diastolic function over follow-up, suggesting that these occur later in the natural history of ALMS cardiomyopathy. Longer term studies should be performed to confirm whether the development of DIF over time may be the primary driver rather than a consequence of ventricular dysfunction and heart failure.

\section{Abbreviations \\ A: Mitral atrial filling velocity; ALMS: Alström syndrome; AR: Autoregressive; BSA: Body surface area; CAC: Coronary artery calcium; CAD: Coronary artery disease; CMR: Cardiovascular magnetic resonance imaging; CTCA: Computed Tomography Coronary Angiography; DIF: Diffuse interstitial fibrosis; E: Mitral early filling velocity; e': Early myocardial relaxation velocity; \\ ECG: Electrocardiogram; ECV: Extracellular volume; EF: Ejection fraction; GBCA: Gadolinium-based contrast agent; GEE: Generalised estimating equation; GLS: Global longitudinal strain; ICC: Intraclass correlation coefficient; ID: Identification number; IQR: Interquartile range; LAV: Left atrial volume; LGE: Late gadolinium enhancement; LV: Left ventricle; EDV: End diastolic volume; EDVi: End diastolic volume indexed to body surface area; ESV: End systolic volume; ESVi: End systolic volume indexed to body surface area; MOLLI: Modified look-locker inversion recovery sequence; NT pro-BNP: N- terminal pro B natriuretic peptide; ROI: Region of interest; RV: Right ventricle; SAX: Short axis; SD: Standard deviation; TTE: Transthoracic echocardiography}

\section{Acknowledgements}

The authors acknowledge the support of Alström society UK (ALMSUK) who have been involved in all stages of the study and instrumental to its success.

\section{Authors' contributions}

RS, TH and NCE conceived the study, reviewed data and contributed to the manuscript; SB, RD, RV and BL collated data, contributed to analysis and to the manuscript; JH provided statistical expertise and analysis; LF reviewed and critically contributed to the manuscript. All authors read and approved the final manuscript

\section{Funding}

No external funding was required but data were collected through the national clinical service.

\section{Availability of data and materials}

The datasets generated and/or analysed during the current study are not publicly available as the single centre location of the study, combined with the nature of the disease, mean that individual patients might be identified through the comprehensive data and material used in this study. These are are available from the corresponding author on reasonable request, subject to discussion on patient identifiable information.

\section{Ethics approval and consent to participate}

This study was approved by local clinical governance commitees and conformed to the principles of Good Clinical Practice. This study utilized clinical data collected in the course of normal patient care (without intention to use it for research at time of collection) and thus does not require ethical approval, consistent with Health Regulation Authority guidance.

Consent for publication

Not applicable.

\section{Competing interests}

The authors declare that they have no competing interests.

\section{Author details}

'Department of Inherited Metabolic Disorders, Queen Elizabeth Hospital Birmingham, Birmingham, UK. ${ }^{2}$ Institute of Cardiovascular Science, University of Birmingham, Birmingham, UK. ${ }^{3}$ Department of Cardiology, Queen Elizabeth Hospital, Birmingham, UK. ${ }^{4}$ Institute of Translational Medicine, Queen Elizabeth Hospital, Birmingham, UK. ${ }^{5}$ Institute of Metabolism and Systems Research, University of Birmingham, Birmingham, UK. ${ }^{6}$ Department of Cardiology, First Floor, Nuffield House, University Hospital Birmingham 
NHS Foundation Trust, Mindelsohn Way, Edgbaston, Birmingham B15 2GW, UK.

Received: 2 April 2020 Accepted: 27 May 2020

Published online: 05 June 2020

\section{References}

1. Warren SE, Schnitt SJ, Bauman AJ, Gianelly RE, Landsberg L, Baim DS. Late onset dilated cardiomyopathy in a unique familial syndrome of hypogonadism and metabolic abnormalities. Am Heart J. 1987;114:1522-4.

2. Marshall JD, Bronson RT, Collin GB, et al. New alström syndrome phenotypes based on the evaluation of 182 cases. Arch Intern Med. 2005; 165:675-83.

3. Corbetti F, Razzolini R, Bettini V, et al. Alström syndrome: cardiac magnetic resonance findings. Int J Cardiol. 2013;167:1257-63.

4. Edwards NC, Moody WE, Yuan M, et al. Diffuse left ventricular interstitial fibrosis is associated with sub-clinical myocardial dysfunction in Alström syndrome: an observational study. Orphanet J Rare Dis. 2015;10:83.

5. Jellis C, Martin J, Narula J, Marwick TH. Assessment of nonischemic myocardial fibrosis. J Am Coll Cardiol. 2010;56:89-97.

6. Puntmann VO, Carr-White G, Jabbour A, et al. Native T1 and ECV of noninfarcted myocardium and outcome in patients with coronary artery disease. J Am Coll Cardiol. 2018;71:766-78.

7. Jatti K, Paisey R, More R. Coronary artery disease in Alström syndrome. Eur J Hum Genet. 2012;20:117-8.

8. Wharton G, Steeds R, Allen J, et al. A minimum dataset for a standard adult transthoracic echocardiogram: a guideline protocol from the British society of echocardiography. Echo Res Pract. 2015;2:G9-G24.

9. Nagueh SF, Smiseth OA, Appleton CP, et al. Recommendations for the evaluation of left ventricular diastolic function by echocardiography: an update from the American Society of Echocardiography and the European Association of Cardiovascular Imaging. J Am Soc Echocardiography. 2016;29: 277-314.

10. Maceira AM, Prasad SK, Khan M, Pennell DJ. Normalized left ventricular systolic and diastolic function by steady state free precession cardiovascular magnetic resonance. J Cardiovasc Magn Reson. 2006;8:417-26.

11. Schelbert EB, Testa SM, Meier CG, Ceyrolles WJ, Levenson JE, Blair AJ. Myocardial extravascular extracellular volume fraction measurement by gadolinium cardiovascular magnetic resonance in humans: slow infusion versus bolus. J Cardiovasc Magn Reson. 2011;13(1):16. https://doi.org/10. 1186/1532-429X-13-16

12. Hundley WG, Bluemke D, Bogaert JG, et al. Society for Cardiovascular Magnetic Resonance guidelines for reporting cardiovascular magnetic resonance examinations. J Cardiovasc Magn Reson. 2009;11:5.

13. Liu B, Dardeer AM, Moody WE, et al. Reference ranges for three-dimensional feature tracking cardiac magnetic resonance: comparison with twodimensional methodology and relevance of age and gender. Int J Cardiovasc Imaging. 2018;34:761-75.

14. Moon JC, Messroghli DR, Kellman P, et al. Myocardial T1 mapping and extracellular volume quantification: a Society for Cardiovascular Magnetic Resonance (SCMR) and CMR Working Group of the European Society of Cardiology consensus statement. J Cardiovasc Magnet Resonance. 2013;15:92.

15. Vijapurapu R, Hawkins F, Liu B, Edwards N, Steeds R. A study of the different methodologies used in calculation of extra-cellular volume by CMR imaging. Heart. 2018;104:A14-A5.

16. Hayer MK, Price AM, Liu B, et al. Diffuse myocardial interstitial fibrosis and dysfunction in early chronic kidney disease. Am J Cardiol. 2018;121:656-60.

17. Dunlay SM, Roger VL, Redfield MM. Epidemiology of heart failure with preserved ejection fraction. Nat Rev Cardiol. 2017;14:591.

18. Cubbon RM, Adams B, Rajwani A, et al. Diabetes mellitus is associated with adverse prognosis in chronic heart failure of ischaemic and non-ischaemic aetiology. Diab Vasc Dis Res. 2013;10:330-6.

19. Baig S, Veeranna V, Bolton S, et al. Treatment with PBI-4050 in patients with Alstrom syndrome: study protocol for a phase 2, single-Centre, single-arm, open-label trial. BMC Endocr Disord. 2018;18:88.

20. Marwick TH, Ritchie R, Shaw JE, Kaye D. Implications of underlying mechanisms for the recognition and Management of Diabetic Cardiomyopathy. J Am Coll Cardiol. 2018;71:339-51.

21. Marshall Jd BRTCGB, et al. New alström syndrome phenotypes based on the evaluation of 182 cases. Arch Intern Med. 2005;165:675-83.
22. Michaud JL, Heon E, Guilbert F, Weill J, Puech B, Benson L. Natural history of Alstrom syndrome in early childhood: onset with dilated cardiomyopathy. J Pediatr. 1996;128(2):225-9.

23. Iles $L$, Pfluger $H$, Phrommintikul $A$, et al. Evaluation of diffuse myocardial fibrosis in heart failure with cardiac magnetic resonance contrast-enhanced T1 mapping. J Am Coll Cardiol. 2008;52(19):1574-80.

24. Bull S, White SK, Piechnik SK, et al. Human non-contrast T1 values and correlation with histology in diffuse fibrosis. Heart. 2013;99:932-7.

25. Sibley CT, Noureldin RA, Gai N, et al. T1 mapping in cardiomyopathy at cardiac MR: comparison with endomyocardial biopsy. Radiology. 2012; 265(3):724-32

26. aus dem Siepen F, Baumgärtner C, Müller-Henessen M, et al. Variability of cardiovascular magnetic resonance (CMR) T1 mapping parameters in healthy volunteers during long-term follow-up. Open Heart. 2018;5:e000717.

27. Yi CJ, Yang E, Lai S, et al. Progression of diffuse myocardial fibrosis assessed by cardiac magnetic resonance T1 mapping. Int J Cardiovasc Imaging. 2014; 30:1339-46.

28. Wong TC, Piehler K, Meier CG, et al. Association between extracellular matrix expansion quantified by cardiovascular magnetic resonance and short-term mortality / clinical perspective. Circulation. 2012;126:1206-16.

29. Schelbert EB, Piehler KM, Zareba KM, et al. Myocardial fibrosis quantified by extracellular volume is associated with subsequent hospitalization for heart failure, death, or both across the Spectrum of ejection fraction and heart failure stage. J Am Heart Assoc. 2015;4.

30. Wong TC, Piehler KM, Kang IA, Kadakkal A, Kellman P, Schwartzman DS. Myocardial extracellular volume fraction quantified by cardiovascular magnetic resonance is increased in diabetes and associated with mortality and incident heart failure admission. Eur Heart J. 2014;35.

31. Puntmann VO, Carr-White G, Jabbour A, et al. T1-mapping and outcome in nonischemic cardiomyopathy: all-cause mortality and heart failure. J Am Coll Cardiol Img. 2016;9:40-50

32. Bond J, Flintoff $\mathrm{K}$, Higgins J, et al. The importance of seeking < em $>$ ALMS1 $1 /$ em $>$ mutations in infants with dilated cardiomyopathy. J Med Genet. 2005;42:e10.

33. Louw JJ, Corveleyn A, Jia Y, labal S, Boshoff D, Gewillig M. Homozygous loss-of-function mutation in ALMS1 causes the lethal disorder mitogenic cardiomyopathy in two siblings. Eur J Med Genet. 2014;57(9):532-5.

34. Shenje LT, Andersen P, Halushka MK, Lui C, Fernandez L, Collin GB. Mutations in Alstrom protein impair terminal differentiation of cardiomyocytes. Nat Commun. 2014;5:3416.

\section{Publisher's Note}

Springer Nature remains neutral with regard to jurisdictional claims in published maps and institutional affiliations.

\section{Ready to submit your research? Choose BMC and benefit from:}

- fast, convenient online submission

- thorough peer review by experienced researchers in your field

- rapid publication on acceptance

- support for research data, including large and complex data types

- gold Open Access which fosters wider collaboration and increased citations

- maximum visibility for your research: over $100 \mathrm{M}$ website views per year

At BMC, research is always in progress.

Learn more biomedcentral.com/submissions 